\title{
A Roadmap towards Service-Oriented Enterprise
}

\author{
Marinela Mircea and Anca loana Andreescu
}

The Bucharest University of Economic Studies, Romania

\begin{abstract}
In the current economic context, enterprises are struggling to obtain competitive advantages on the market, by exploiting the opportunities offered by information technology. The research in this paper aims to encourage the use of modern approaches in order to increase enterprise agility, through the transition to Service-Oriented Enterprise (SOE). The proposed solution is based on reaching a superior level of maturity of Service-Oriented Architecture (SOA) within the organization. SOE integrates the latest technologies with service-oriented business processes within an approach which requires the creation of an agile architecture and also major changes at organizational level. Structured in two main parts, the paper first highlights the SOA maturity levels in organization according to several factors, as well as the knowledge of the impact and the changes arising from SOA adoption. In addition, an architectural model for service-oriented enterprise is proposed, based on up-to-date technologies like SOA, Cloud Computing, Business Process Management and Case Management. This model is intended to facilitate the interoperability between business partners as well as the alignment of information technology strategy and business strategy, and ultimately to increase enterprise agility.
\end{abstract}

Keywords: service-oriented enterprise, enterprise agility, service-oriented architecture, business process management.

\section{Introduction}

The paper will provide for the economic organizations a solution to increase agility in knowledge-based society. The solution is based on reaching a superior level of maturity of service-oriented architecture (SOA) within the organization and its transition to service-oriented enterprise (SOE). The research aims to encourage the use of modern approaches regarding the growth of agility and to bring further knowledge regarding SOE.

SOA is vital to an enterprise as an architecture paradigm because it allows the business to become agile and respond rapidly to changes and challenges it faces internally and externally from its customers, stakeholders and various federal mandates in order to accomplish mission critical objectives (Mansukhani, 2005; Birekul and Dogerlioglu, 2011). A large number of specialists admitted that SOA offers numerous benefits to the organization. Here are some of the key benefits that an enterprise can achieve from SOA (Mansukhani 2005): - agility to collaborate (SOA provides ability to securely and easily share information, with partners and stakeholders); - agility to adapt to market (SOA promotes the ability to rapidly reconfigure the business process); reduction of cost; - improvement in efficiency (SOA promotes a modular enterprise, promising a high degree of

Copyright (C) 2012 Marinela Mircea and Anca Ioana Andreescu. This is an open access article distributed under the Creative Commons Attribution License unported 3.0, which permits unrestricted use, distribution, and reproduction in any medium, provided that original work is properly cited. Contact author: Marinela Mircea Email: mmircea@ase.ro 
reusability of business services, ensuring consistency); - better business operations; ease of introducing new technologies.

The tendency of SOA maturity growth observed within the past years inside organizations leads to new types of enterprises, namely service-oriented enterprises. SOE involve the application of service orientation in all main operations and management of the enterprise, including human resources, business processes, information systems and decisions management. Also, SOE exceed the SOA promises to increase agility, providing vision on demand. This requires a superior level of SOA maturity, agile architectures and major effects at the organizational level. SOE integrates the latest technologies with service-oriented business processes within an approach in which each participant can be seen as a provider and as a consumer of services. Service orientation helps organizations to lower information technology-related costs while making information technology flexible enough to deliver what they really need (i.e. simplicity, agility, and value). Also, it enables organizations to achieve agility so they are able to respond quickly and easily to change, helping to maximize return, mitigate risk, improve performance and increase agility (Mansukhani, 2005).

At present, there are a limited number of organization architectures, architectures of systems of applications and performance analysis for service-oriented environments. In addition, transition/development methodologies, performance analysis and optimization models for service-oriented enterprises/networks of enterprises are rare and not very systematic. Due to the important changes generated by the transition to SOE, a significant step for SOA success would be the analysis of the SOA maturity level in organization, as well as the knowledge of the impact and the changes arising from the SOA adoption. The creation of an architectural model is also of great importance. It might lead to the alignment between business and information technology to produce an agile architecture of the SOE.

\section{Service-Oriented Architecture Maturity}

The transition to SOE leads to changes in the entire organization, considered to be a long term transition process. Birekul and Dogerlioglu (2011) state that SOA is a strategy which requires long-term commitment and will take several years to be established properly. Organizations generally use an incremental implementation in adopting SOA. Initiation is achieved by small projects in one or several departments. Extending and adopting a superior level of SOA maturity in organization depends on the projects' success and the results obtained.

An important step in the transition to SOE is the analysis of the organization's SOA maturity. At present, there are several classifications of the organization depending on SOA maturity. Based on the analysis of different classifications (PWC, 2009; Pugsley, 2006; IMS, 2009) we suggest the following six levels of SOA maturity in the organization (figure 1): (1 initiation, 2 experimenting, 3 integration, 4 standardization, 5 selfmanaged and $\boldsymbol{6}$ adaptive. This classification takes into account both the localization of SOA at organization level as well as the influence/lining up of SOA with the organization's strategy. The real benefits of SOA will be achieved when the organization stops investing locally and starts investing in the whole organization. 


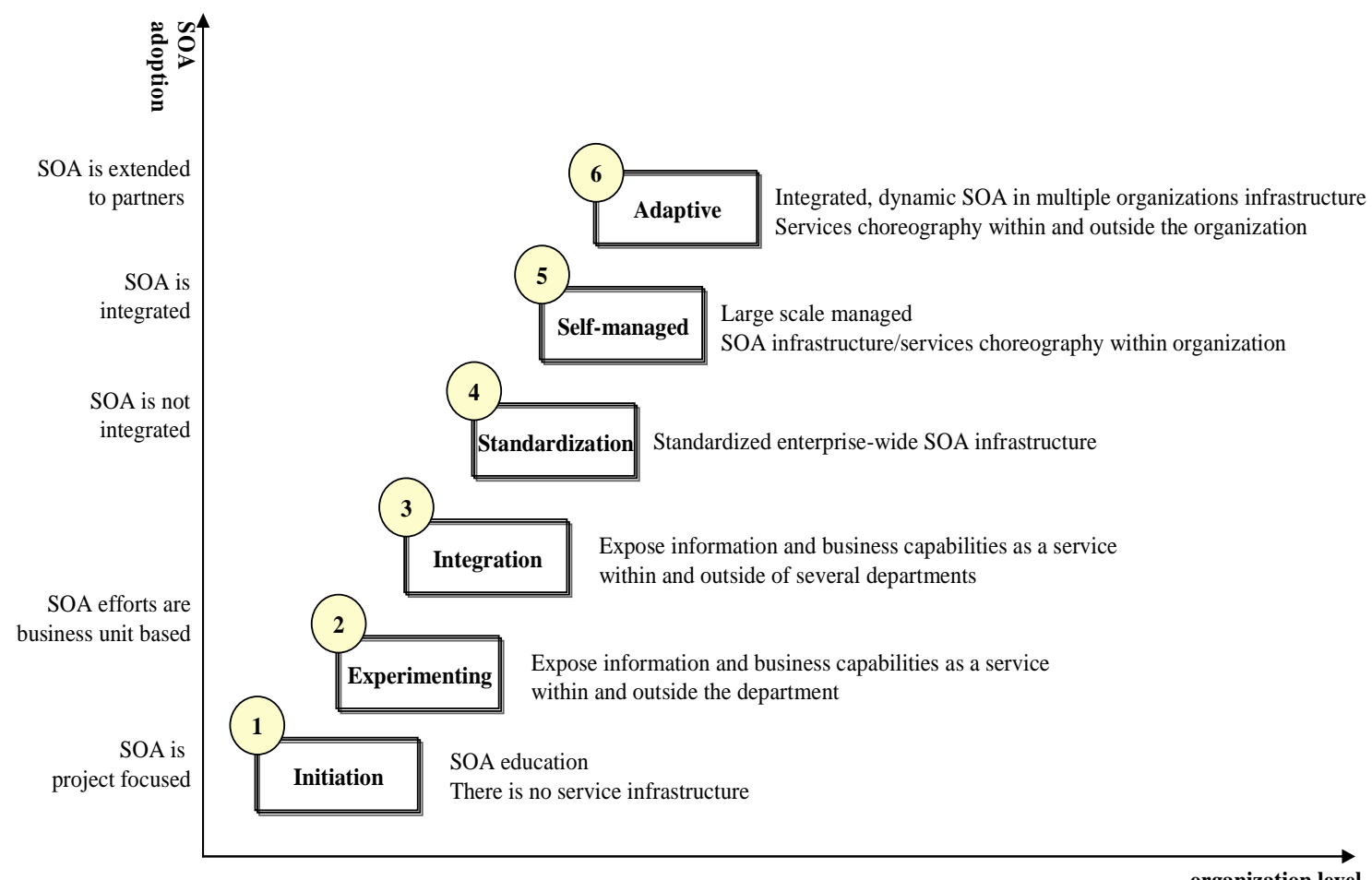

Stage 1: Initiation. At this stage the organization has minimum knowledge about the SOA or this aspect totally lacks. The management's interest for SOA is also minimal and centered on projects, not on business.

Stage 2: Experimenting. This phase starts off by exposing information and business capabilities as a service inside the department and then outside the department. The organizations that find themselves at this stage achieve the SOA benefits through the ROI (Return On Investment) indicator.

Stage 3: Integration. At this stage the expansion of SOA takes place at the level of several departments so as to facilitate the cooperation and improvement of the processes. The SOA is centered on business units and is understood only by a part of the IT (Information Technology) staff and management.

Stage 4: Standardization. In this phase, the SOA is extended at the organization level in order to facilitate the cooperation and improvement of business processes, but it is not integrated. The organization continues to align business processes with technology in order to obtain performance with respect to the reuse of the services and changes imposed by the business environment.

Stage 5: Self-managed. At this stage SOA is integrated at the organization level and organizations may respond proactively to the market changes. Additionally, the organization performance is also measured by service-oriented indicators which measure the positive impact of the SOA upon the organization.

Stage 6: Adaptive. At this stage, SOA is fundamental for all the important operations (internal and with business partners) and for business management. Therefore, the enterprise becomes service-oriented. According to Graves (2009), within a serviceoriented enterprise everything is a service and the enterprise itself is a service, creating new space for agility and innovation for the enterprise. In a service-oriented enterprise, 
ROI will indicate the continuous improvements in achieving the organization's objectives.

Currently, a large number of organizations find themselves within the first stages of SOA maturity. According to a survey performed with experts in SOA development (34 professionals from Turkey and several European countries including Denmark, Greece, Italy, Poland, Romania, and the Slovak Republic), the stage of SOA in the respondents' organization are (Birekul and Dogerlioglu, 2011): in development (18\%), deployed in a single department (18\%), deployed in multiple departments $(50 \%)$ and deployed for external use (14\%). The achievement of a high SOA maturity level does not represent a purpose in itself and it must align with the organization strategy. Furthermore, the opportunity of passing on to SOA, depending on several influence factors, was analyzed and depicted in Table 1.

Table 1: Service-oriented Architecture Necessity

\begin{tabular}{|l|l|}
\hline Influence factor & \multicolumn{1}{|c|}{ SOA necessity } \\
\hline $\begin{array}{l}\text { Nature of business } \\
\text { processes }\end{array}$ & $\begin{array}{l}\text { The decision of passing on to SOA may be taken if there is a possibility of } \\
\text { automating a sufficient number of business processes or of renouncing when } \\
\text { processes are generally manual and without high possibilities of automation. }\end{array}$ \\
\hline $\begin{array}{l}\text { The existence of } \\
\text { financial } \\
\text { resources }\end{array}$ & $\begin{array}{l}\text { The transition to SOA depends on the percentage of the organization's budget } \\
\text { allocated to IT integration activities. }\end{array}$ \\
\hline $\begin{array}{l}\text { Integration level } \\
\text { of existent } \\
\text { systems }\end{array}$ & $\begin{array}{l}\text { In the situation in which there are applications that do not need integration or } \\
\text { need a low integration level, the decision of renouncing at the extension of } \\
\text { SOA may be taken. }\end{array}$ \\
\hline $\begin{array}{l}\text { Collaborative } \\
\text { environment }\end{array}$ & $\begin{array}{l}\text { The need of creating an interaction with the partners and their effectiveness } \\
\text { leads to the necessity of using SOA. }\end{array}$ \\
\hline
\end{tabular}

The transition to SOE may be performed through different types of approaches. Choosing the type of approach depends on the organizational requirements and its alignment to the organization's strategy. The adoption of SOA may be done incrementally by using one of the following types of approaches (Narayanan, 2010): top-down, button-up, and mixed (this approach presents a more decreased risk of SOA integration). Whatever approach is chosen, the transition to SOE depends on a variety of factors. We will identify the main factors starting from the fact that the organization must continuously control the dynamics of the evolution of the business processes, information systems and human resources. Business agility may also be obtained by combining the business processes agility with information technology agility and human resources agility (El-Ghareeb, n.d.). Thus, the adoption of SOA will be analyzed depending on the three factors: business processes, information technology and human resources. In this respect, SOA objectives are: coupling business processes, decoupling information technology and obtaining staff agility.

\section{Business Processes}

One of the factors that influences business processes passing on to SOA is the complexity of business processes and their documentation level. The business model represents an important factor on which the success of 
moving on to SOA depends. Considering the global context generated by the digital economy and by the complexity of interacting with partners, one might say that business processes evolved from those pertaining a) to a department, b) to interdepartmental processes between various branches of the organization and c) to organization networks from the same country or different countries. The existence of some collaborative processes that imply complex interactions between participants and the necessity of using knowledge leads to an increased complexity of business processes and, afterward, to the complexity of implementing SOA. A complete documentation of business processes and their understanding represent a key element in describing the services and the success factor for the transition to SOA. An increased degree of complexity combined with a low level of process documentation leads to challenges/problems in moving on to SOA and sometimes it may lead to the decision of giving up.

Another factor that influences the adoption of SOA is the possibility of processes automation. Finding itself in all business processes, knowledge represents a performance indicator for the organization. Business processes tend to be integrated with knowledge and business intelligence in order to obtain agility in the collaborative environment (Mircea, Ghilic-Micu and Stoica, 2010). The existence of some processes intensively based on knowledge (especially decisional processes) determines, at the level of processes, the automation of a subset of activities only. The number of manual processes or of those that can be less automated within an organization may represent an important factor to be considered when deciding to adopt SOA.

A third factor that may be taken into account in the analysis of business processes is the service orientation of business processes. Within the last few years, the combined use of some instruments of business processes management (BPM) with SOA led to a new development stage of business processes, namely service-oriented business processes. Service-oriented business process includes, apart from the classical elements of a business process, also network services, which are services that had implemented certain business-process functions and had been capsulated and published on network (Zeng, Huang and Fan, 2007).

\section{Information Technology}

The approach types, as well as the rapidity of integrating SOA depend on the existence of informational systems within the organization, their heterogeneity and the technology type used. It is also important to identify the existent infrastructure and the way they interact and communicate between themselves. For this reason, an analysis concerning the integration/migration strategies and their costs must be performed when passing on to SOA (Mircea and Andreescu, 2009).

\section{Human Resources}

The success of adopting SOA depends on the flexibility of human resources and communication within the organization. An increased level of the human resources agility represents a stimulating factor for the success of SOA. Agility of human resources can be achieved through an organizational culture that provides a workforce intensively based on technological and business knowledge in all areas of business (Mircea, 2011). The organizational culture must allow rapid adaptation of labor to the continuously changing business environment.

Multidisciplinary teams, with members from both IT and business domains were seen as crucial for SOA development (Antikainen and Pekkola, 2009). Knowledge about the business strategy has an important role in designing reusable services and architectures that would fit future business needs without major re-implementation efforts. According to Antikainen and Pekkola (2009), business stakeholders may contribute to this by 
interpreting and clarifying the models for the IT developers, participation in the project's management change, exception control and ensuring the congruence of SOA implementation in the long run. At the same time, more and more accent is placed on the employees that have knowledge in domains related to the activity they carry out. Knowledge workers attract more and more the attention of managers because their percentage in organizations is increasing (between 25\% and 50\%; Swenson, 2010) and their wages are high.

The transition to SOE supposes changes in the whole organization. Some of the most important changes are the following:

* Organization culture. According to a survey elaborated in several governmental agencies (Rogers, 1996) the shift "from a control-oriented bureaucracy to one of service to the public, combining better quality of service with demanding financial and performance targets" takes place. The most important changes identified according to the data reported by those six agencies were: focus on financial (bottomline) performance, focus on service quality, and focus on public service purposes. At the same time, the policy and practices of the organization must be aligned in order to increase the quality of SOA implementation along with the objectives of the business. These must lead to a change of the organization culture, from a conservative culture, of control and command to an adaptive organizational culture.

* Management. Aligning the staff to SOA implies aligning the managerial staff with the management systems. Managers must have capabilities that allow them to perform a proactive management based on the data supplied by the informational system of the organization. A change takes place at the same time - many processes, especially the decisional ones are based on knowledge. The changes within the structure of the organization depend on the existing structure of the organization. According to a study performed by Birekul and Dogerlioglu (2011) with 34 experts in SOA the need for a structural change within the questioned organization was (positive/negative responses): functional yes: 10/ no: 2; divisional - yes: 11/ no: 2; matrix - yes: 9/ no: 0. Adopting SOA implies a decentralized control at the level of the organization (Malone, 2004).

\%outsourcing. SOA offers a type of architecture favorable to outsourcing. This way, organizations may standardize their business processes and externalize those processes that cannot be found on the value chain. SOA facilitates the organization's orientation on its competences and the externalization of the other business processes/tasks, subtasks. SOA adoption requires organizations to outsource more than before and business units to move part of operations to customers and suppliers (Birekul and Dogerlioglu, 2011). It also facilitates the diminishing of the outsourcing risk. This may be accomplished through outsourcing sub-processes or tasks witch can reduce the risk of loosing strategic advantages. In the study (Benazeer et. al., 2004), the authors demonstrate the positive impact of SOA upon outsourcing, with references to the main publications on this aspect. Some examples of positive impact of SOA are: standard processes allow easier outsourcing of process capabilities; technical dependence can be reduced through SOA; - SOA increase competition among providers which can result in reduced cost of outsourcing and improved quality of service; - virtual communication between organizations will help companies to decide what to keep in-house and what to outsource; - SOA provides an abstraction layer on top of the existing technology resources, allowing third parties to provide those resources more easily.

* Business processes. Adopting SOA implies decomposing the key processes into sub- 
processes, discreetly and slightly interconnected. The deconstruction process converts an enterprise into a set of automatic and small components (business components) which interact with the similar entities in the business of ecosystem (Tohidi, 2011). Thus, the business is seen as a set of interdependent services which create elementary tasks. The management of these types of business processes will be done in accordance with the principles of abstraction, specialization and separation of concerns in addition to the flexibility that service-oriented architecture promotes (Mircea, 2010).

\section{An Agile Architecture for Service-Oriented Enterprise}

Obtaining the agility and the competitive advantage implies the creation of an architecture that aligns the capabilities of informational systems with the business objectives. For an enterprise, agility represents its ability to respond and adapt to change. Presently, agility is considered one of the most important factors of the organization, allowing it to provide efficient services to the clients and to obtain competitive advantage on the market. This may be achieved by aligning information technology to the business so that it supports the necessities, objectives and strategies of the organization. This section comes to support the answers for the following questions:

- Which are the modern approaches that may be used in creating an agile architecture of SOE?

- How and which one of these approaches may help the achievement of the alignment between business and information technology?

SOE architecture may integrate several modern approaches for creating the optimum solution of aligning the business with information technology. SOE engineering further develops the enterprise engineering approach by selecting service as governing paradigm (Nurcan and Schmidt, n.d.). Based on the analysis of the actual stage regarding the creation of architectures, we suggest the creation of an agile architecture for SOE using the following approaches: SOA, Cloud Computing, BPM and Case Management.

SOA facilitates the definition of the services reflecting business capabilities and informational necessities of the organization. SOA provides flexible software components that allow organizations to quickly adapt to the permanent demands of market changes. SOA has the advantage of producing simple, modular, loosely coupled components, therefore allowing for quick re-composition of the applicative frame of functionalities that they provide. The technological agility can be obtained through SOA.

Cloud Computing, just like SOA, uses the service like a structuring paradigm. It is considered the next step in the evolution of the Internet, providing to the organization the means of using IT services. As enterprise applications grow in complexity, the need for organizations to leverage cloud computing as part of their SOA platform is growing exponentially. Combining SOA with cloud computing prepares businesses for the next generation of flexible, distributed and scalable cloud-enabled business applications, while facilitating greater operational efficiency, generating more revenue, and helping businesses enter new markets. The essential characteristics for cloud computing are on-demand self-service, broad network access, resource pooling, rapid elasticity, and measured service (Behrendt et. al., 2011). Even if these characteristics can be found in SOA requirements and solutions in various organizations today, they are optional for SOA.

Cloud and agile concepts have some common characteristics: both terms mean able to move quickly, with skill, and control. Cloud computing applications are highly automated. With most providers, the 
software is already developed, installed on the machines, and ready for all the users. Cloud computing and agile development are also complementary concepts that have come together in myriad ways to aid in the rapid development and deployment of software to meet real business requirements (Dooley, 2010). The association of agile with cloud leads to fast improvements with minimum costs.

$B P M$ contributes at the development of the organization's architecture, helping to identify the main processes of the organization and business capabilities. BPM is the core layer of any SOE architecture, helping at the alignment of processes with information technology. BPM is considered to be a proactive instrument, allowing the organization to measure performance and make the necessary adjustments. Another advantage of BPM is the documentation of the processes, which finally leads to the rationalizing of business processes. BPM promotes agile and collaborative execution, through the offer of flexible business processes. A process is automated by BPM and it becomes a reusable service.

With the focus on enterprise agility, BPM provides a lifecycle of improvement to enable agility and the ability to establish process improvement. BPM and SOA enhance each other bidirectional (Chow, Medley and Richardson, 2007): SOA enables both the faster deployment of BPM and the enablement of its reusability; BPM provides orchestration, control, and governance of the SOA infrastructure. BPM's and SOA's impact on agility is more than the sum of their parts (because both the methodologies and technologies of BPM and SOA are compatible).

Case Management is considered a very used BPM pattern. While case management offers many of the BPM benefits, it is meant to a different class of problems, namely ad hoc processes, dynamic and unstructured (Shepherd, n.d), non-repetitive, unpredictable, emergent, robust in front of varying conditions (Swenson, 2010), and intensively based on knowledge. The automation of case management is necessary in order to make activities more efficient, to eliminate human errors, and to diminish the expenses with training and memorizing the knowledge about processes. Case-oriented BPM is considered the best automation solution of case management (Singularity, 2009). A dynamic case management approach allows the valorization of the advantages of all existing resources including people, processes and systems, achievement of better service outcomes and increased business agility. Essentially, this approach is taking into account the importance of knowledge within organization and the existence of processes intensively based on knowledge.

The use of the approaches mentioned above helped to create a connection between the objectives and the strategy of the organization and its resources and led to the design of an enterprise architecture. The enterprise architecture (figure 2) is serviceoriented, and it serves at the same time at eliminating the gap between the business level and the information system level. 


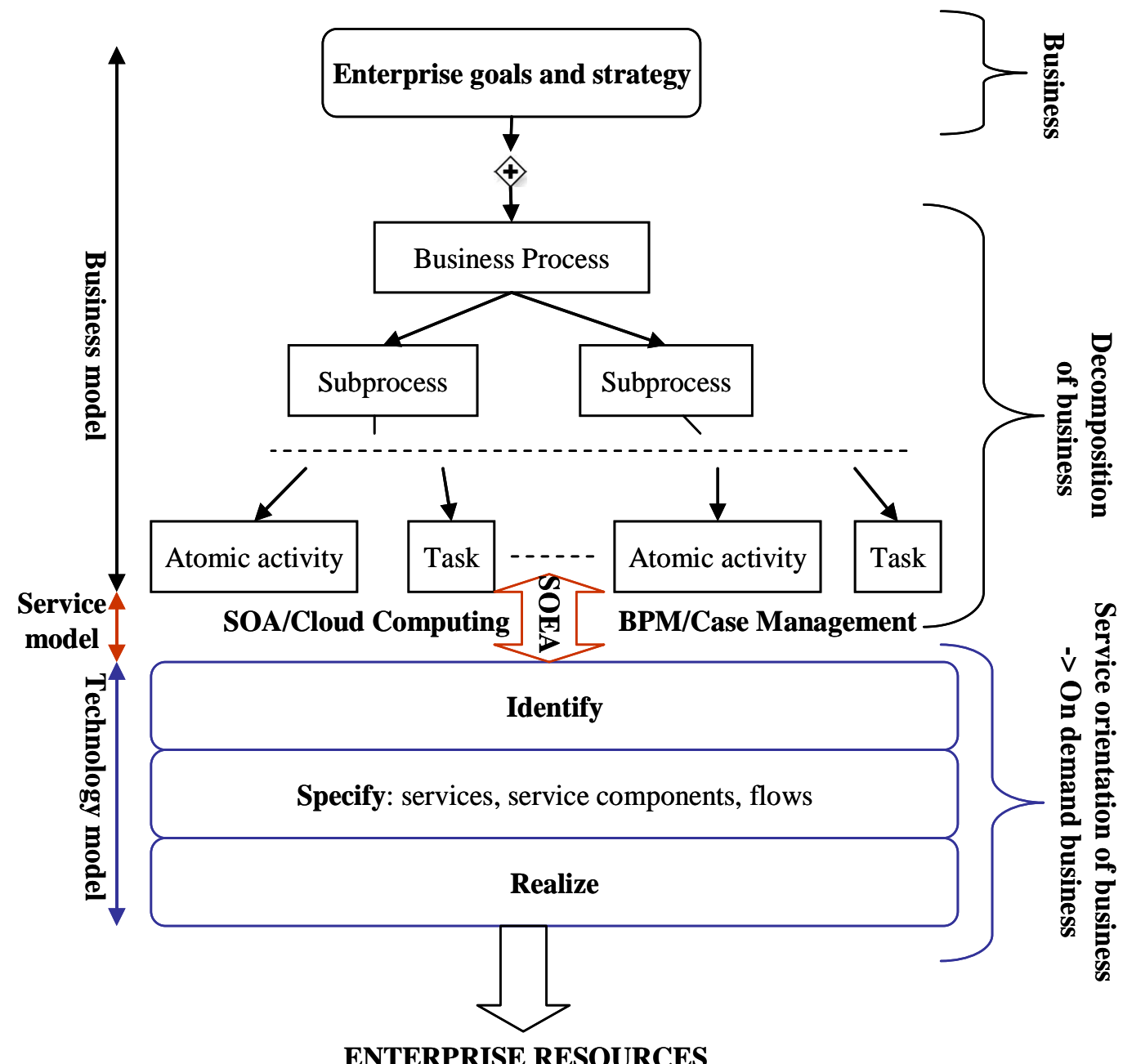

SOE architecture allows alignment at strategic level (aligning the business strategy with the IT strategy), at the planning level (reducing costs, establishing the investment decisions, optimization of ROI indicator) and at the designing level (infrastructure for business requirements). The proposed architectural model is based on the alignment offered by the principles, practices of the previously mentioned approaches, and may be described in three steps (table 2):
- $\quad$ Step 1 - describing and decomposing the business starting from the strategy and objectives of the business;

- $\quad$ Step 2 - identification, description and realization of business services;

- $\quad$ Step 3 - governance of service-oriented enterprise architecture. 
Table 2: The Steps of the Proposed Model

\begin{tabular}{|c|l|c|}
\hline Step & \multicolumn{1}{|c|}{ Techniques /standards } & Results \\
\hline 1 & - Business Performance Management, Component Business Model \\
& - Business Process Maturity Model, Business Process Modeling Notation, \\
& Business Process Definition Metamodel & $\begin{array}{c}\text { Elementary } \\
\text { tasks }\end{array}$ \\
\hline 2 & $\begin{array}{l}\text { Unified Service Model, Business Reference Model (Nugent, 2004) } \\
\text { - Semantic of Business Vocabulary and Business Rules, SOA Modeling } \\
\end{array}$ & $\begin{array}{c}\text { Business } \\
\text { services }\end{array}$ \\
\hline 3 & -SOA, Cloud Computing, Service Reference Model & $\begin{array}{c}\text { SOE } \\
\text { architecture }\end{array}$ \\
\hline
\end{tabular}

The three steps suppose the use of techniques and/or standards that lead to the accomplishment of SOE architecture. The first step refers to the use of techniques/standards regarding processes; the second step refers to techniques/standards useful for the identifications, description and accomplishment of services; the last step refers to useful approaches in the accomplishment of SOE architecture. The achievement of each step also leads to certain results: step 1- the elementary tasks/activities of the business; step 2 - the business services identified and described in order to achieve the elementary tasks/activities of step 1; step 3 - the SOE architecture that shall provide the necessary resources of the organization in order to attain its objectives.

As far as step 3 is concerned, the SOE architecture governance contains both information technology processes necessary to assure the correctness and quality of services, as well as the organizational processes that assure that the organization stakeholders will receive the added value by adopting SOA. Consequently, the SOA governance must also assure the alignment between the information technology governance and the organizational governance. Generally, SOA governance has an incremental increase directly proportional with the increase of the SOA maturity level in the organization. Just like SOA, it must correspond with the needs of the organization and not be seen as a purpose in itself.
As far as the SOE architecture is concerned, SOA offers alignment between the business processes and informational systems at the level of architecture, governance and communication (Chen, 2007). The three levels of alignment may be combined in order to create an alignment strategy that leads to innovation. Also, SOA offers support in creating and managing shared services. Cloud computing is helping organizations move towards an open and collaborative environment while reducing the costs of delivering and managing the needed services. The use of the cloud computing approach leads to a type of architecture, which is more business-aligned, governance-oriented, and less technology-oriented. From a business perspective, the real potential of cloud computing is that it can improve the alignment between information technology and business (Mitchell, n.d.).

As information technology strategy and business strategy become increasingly aligned, the business community's involvement in managing information assets will grow in importance. IT teams will concentrate more in defining the information architecture, in managing its information assets, and in making sure these information assets are properly aligned to the business strategy and objectives.

\section{Conclusions}

In the conditions of an environment characterized by permanent changes, externalization of activities and globalization, organizations and business in general strive 
to value to the maximum the opportunities offered by information technology in order to obtain a competitive advantage on the market. One of the successful factors leading to development is increasing the organization's agility. The paper confers an important role to service-oriented architecture in the process of passing on to service-oriented enterprise and increasing enterprise agility. Considering that the simple adoption of information technology in the organization does not guaranty economic success, an analysis of the success in aligning information technology to the business requirements must be made.

From the point of view of the aspects presented, a service-oriented enterprise may be a feasible response to the challenges of the economic and business environment. Through such a solution, the economic organization - small, medium or large may speculate market opportunities, which in the lack of agility, may not be accessed. Thus, by resource partitioning and increase of technology integration speed, by making more dynamic the business processes, transition to SOE may be an adaptation response of organizations to the present socio-economical conditions.

\section{Acknowledgments}

This work was supported by CNCSISUEFISCSU, project PN II-RU (PD), code 654/2010, contract no. 12/03.08.2010.

\section{References}

Antikainen, J. \& Pekkola, S. (2009). "Factors Influencing the Alignment of SOA Development with Business Objectives," LSE Department of Information Systems. [Online], [Retrieved December 10, 2011], http://is2.lse.ac.uk/asp/aspecis/20090213.p df

Behrendt, M., Glasner, B., Kopp, P., Dieckmann, R., Breiter, G., Pappe, S., Kreger, H. \& Arsanjani, A. (2011). 'Introduction and Architecture Overview IBM Cloud Computing Reference Architecture 2.0.,' IBM 2011. 1-36.
Benazeer, S., Verelst, J., Grembergen, V. W. \& Mannaert, H. (2004). 'Aligning Technology with Business an Analysis of the Impact of SOA on Outsourcing,' Journal of Theoretical and Applied Information Technology, 4 (3), 244-252.

Birekul, I. \& Dogerlioglu, O. (2011). "Impacts of Service-Oriented Architecture Transformation on Organizational Structures," Journal of Applied Sciences, 11(15), 1-9.

Chen, H.- M. (2007). "Enterprise Architecture and Business-IT alignment," Infwest. [Online], [Retrieved December 10, 2011], https://www.jyu.fi/it/laitokset/cs/en/infwe st/hongmei

Chow, L., Medley, C. \& Richardson, C. (2007). "BPM and Service-Oriented Architecture Teamed Together: A Pathway to Success for an Agile Government," TechRepublic. [Online], [Retrieved December 10, 2011], http://www.techrepublic.com/whitepapers/ bpm-and-service-oriented-architectureteamed-together-a-pathway-to-success-foran-agile-government/342112

Dooley, B. J. (2010). "Cloud Implications for Agile Development," Cutter Consortium. [Online], [Retrieved December 10, 2011], http://www.cutter.com/content/project/full text/reports/2010/05/index.html

El-Ghareeb, H. A. M. (n.d.). "Aligning Service Oriented Architecture and Business Process Management Systems to Achieve Business Agility," [Online], [Retrieved December 10, 2011],

http://www.hfgilbert.com/rc/Whitepapers/ ServiceOrientedArchitecture.pdf

Graves, T. (2009). The Service-Oriented Enterprise, Tetradian Books, England.

IMS. (2009). "Adoption of Service Oriented Architecture for Enterprise Systems in Education: Recommended practices," IMS Global Learning Consortium, [Online], [Retrieved December 10, 2011], 
http://www.imsglobal.org/soa/soawpv1p0/ imsSOAWhitePaper_v1p0.pdf

Luftman, J. (2003). "Measure Your BusinessIT Alignment," Optimizemag, 76-80. [Online], [Retrieved December 10, 2011], http://www.bita-

center.com/pdf/Alignment_Maturity_Summa ry.pdf

Mansukhani, M. (2005). "Service Oriented Architecture White Paper," [Online], [Retrieved December 10, 2011], http://h71028.www7.hp.com/services/dow nloads/soa_wp_062005.pdf

Mircea, M. (2010). "Adapt Business Processes to Service Oriented Environment to Achieve Business Agility," Journal of Applied Quantitative Methods, 5 (4).

Mircea, M. (2011). "Service-Oriented Enterprise: Taking the Next Step beyond Agility in the Digital Economy," Proccedings of the International Conference on Digital Enterprise and Information Systems 2011, July 20-22, London, UK.

Mircea, M. \& Andreescu, A. I. (2009). 'Architecture Reengineering for Services Oriented Businesses,' Journal of Applied Collaborative Systems, 1(2), 55-66.

Mircea, M., Ghilic-Micu, B. \& Stoica, M. (2010). 'Combining Knowledge, Process and Business Intelligence to Delivering Agility in Collaborative Environment,' L. Fischer (Ed.) 2010 BPM and Workflow Handbook, Spotlight on Business Intelligence (pp. 99114). Florida, USA: Future Strategies.

Mitchell, D. (n.d.). "Cloud Computing. and the Goal of IT-Business Alignment," Forbes Custom. [Online], [Retrieved December 10, 2011],

http://www.forbescustom.com/TechnologyP gs/CloudComputingP1.html

Narayanan, V. (2010). 'Modern SOA Methodology and SOA Adoption Using Agile Practices (Part I),' SOA Magazine, (42), 1-6.
Nugent, M. (2004). "The Four Phases of IT/Business Alignment," It Business Edge. [Online], [Retrieved December 10, 2011], http://www.cioupdate.com/insights/article. php/3446591/The-Four-Phases-of-

ITBusiness-Alignment.htm

Nurcan, S. \& Schmidt, R. (n.d.). "For Engineering Service-Oriented Enterprises in the Era of Cloud Computing. Could EA Notations be a Lingua Franca or Modern Hieroglyphs?," SOEA4EE. [Online], [Retrieved December 10, 2011], http://www.soea4ee.org/

Plazaola, L., Molina, E. S., Vargas, N., Flores, J. \& Ekstedt, M. (2006). "A Metamodel for Strategic Business and IT Alignment Assesment," [Online], [Retrieved December 10, 2011], http://www.asdifec.uni.edu.ni/Sitio/Norman Vargas/187-Plazaola-

A\%20METAMODEL\%20FOR\%20STRATEGIC $\%$ 20BUSINESS $\% 20$ AND\%20IT\%20ALIGNME NT\%20ASSESMENT.pdf

Pugsley, A. (2006). "Assessing Your SOA Program," HP. [Online], [Retrieved December 10, 2011], ftp://15.192.45.22/pub/services/soa/info/4 AA0-4824ENW.pdf

PWC. (2009). "Achieving Business-Driven SOA. Point of View," PricewaterhouseCoopers. [Online], [Retrieved December 10, 2011], http://www.pwc.com/en_US/us/increasingit-effectiveness/assets/business-drivensoa.pdf

Rogers, B. (1996). "Special Operating Agencies: Human Resources Management Issues," Management Practices, 14, 1-35.

Shepherd, T. (n.d). "Top Case Management Misconceptions," Global 360. [Online], [Retrieved December 10, 2011], http://www.global360.com/xres/uploads/re source-centerdocuments/021210_WhitePaper_CaseMgmt Misconceptions.pdf 
13 Journal of Organizational Management Studies

Singularity. (2009). "Case Management: Combining Knowledge With Process," BPTrends.

Swenson, K. (2010). 'The Nature of Knowledge Work,' Workflow Management Coalition. [Online], [Retrieved December 10, 2011], http://www.wfmc.org/webinar-oncase-management-for-knowledge-work.html

Tohidi, H. (2011). "Modeling of Business Services in Service Oriented Enterprises," Procedia Computer Science (3), 1147-1156.

Zeng, S., Huang, S. \& Fan, Y. (2007). "ServiceOriented Business Process Modeling and Performance Evaluation based on AHP and Simulation," Proceedings of ICEBE 2007 , Hongkong, October 24-26. 